PACCO HC; RINALDI MM; SANDRI D; NEVES PHC; VALENTE RRM. 2014. Características de tomate producido con agua tratada en interior y exterior de invernadero. Horticultura Brasileira 32: 417-425. DOI - http://dx.doi.org/10.1590/S0102-053620140000400008

\title{
Características de tomate producido con agua tratada en interior y exterior de invernadero
}

\author{
Honorato C Pacco ${ }^{1}$; Maria M Rinaldi²; Delvio Sandri ${ }^{3}$; Pedro HC Neves*; Ricardo RM Valente*

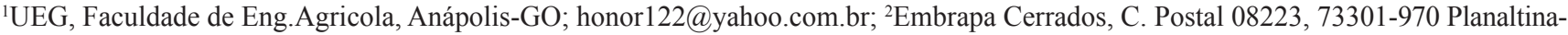 \\ DF; madalena.rinaldi@embrapa.br; ${ }^{3} \mathrm{UnB}-\mathrm{FAMV}$, Brasilia-DF; sandri@unb.br, "bolsistas CNPq'
}

\section{RESUMEN}

El objetivo de este trabajo de investigación fue determinar el rendimiento y calidad post-cosecha de tomate "Dominador" producido en el interior y exterior de invernadero, irrigado por el sistema de goteo superficial y sub-superficial con agua tratada y agua natural de pozo. El planeamiento experimental fue el de bloques casualizados $3 \times 2$, siendo las parcelas constituidas por la calidad de agua agua residual, agua para ferti-riego convencional y agua natural, con 4 repeticiones. No hubo efecto significativo en el interior y exterior del invernadero sobre la productividad, la calidad de frutos, excepto en la relación de sólidos solubles/acidez de titulación, donde el mayor valor de 2,46 , fue para cultivados en el interior del invernadero, y con incremento significativo durante el período de almacenamiento (catorce días) que fue de $102 \%$, lo que indica una calidad superior durante el período de almacenamiento. Los valores de $\mathrm{pH}$ de los frutos producidos en el interior y exterior del invernadero durante el periodo de almacenamiento indicaron como frutos ácidos. Los sólidos solubles se elevó y la acidez titulación se redujo durante el periodo de almacenamiento para todos los tratamientos. El tomate presentó vida útil post-cosecha de siete días para todos los tratamientos en ambos ambientes de cultivo y almacenamiento a $27^{\circ} \mathrm{C}$ y $60 \%$ de humedad relativa.

Palabras clave: Lycopersicon esculentum, post-cosecha, irrigación, re-uso, vida útil.

\begin{abstract}
Features tomato wastewater produced inside and outside the greenhouse

The objective of this research was to determine the yield and postharvest quality of tomato "Dominador" produced inside and outside the greenhouse, irrigated by the system of surface and subsurface drip with wastewater and natural water. The experimental design was a randomized block $3 \times 2$, the portions being formed by the water quality (wastewater, water for conventional fertigation and natural water), with four repetitions. There was no significant effect of cultivars within and outside the greenhouse on the productivity index, fruit size and quality, except in the ratio of soluble solids/ titratable acidity, where the highest value (2.46) was for growing inside the greenhouse, and significant increases (102\%) during the storage period (fourteen days), indicating a superior quality during the storage period. The $\mathrm{pH}$ of the fruits produced within the greenhouse ranged from 3.95 to 4.31 , and values for tomatoes produced outside the greenhouse ranged from 4.00 to $4.22 \mathrm{in} \mathrm{pH}$ during the storage. The soluble solids increased and titratable acidity was reduced during the storage period for all treatments. The tomato had shelf-life of seven days for all treatments in both cultivation environments, and stored at $27^{\circ} \mathrm{C}$ and $60 \%$ relative humidity.
\end{abstract}

Keywords: Lycopersicon esculentum, post-harvest, irrigation, reuse, life.

(Recebido para publicação em 30 de janeiro de 2013; aceito em 1 de agosto de 2014)

(Received on January 30, 2013; accepted on August 1, 2014)

$\mathrm{E}^{\mathrm{n}}$ todo el mundo, crece la conciencia en torno de la importancia del uso racional del agua, de la necesidad de control de pierdas, desperdicios y de la introducción definitiva de reciclaje de agua para atender los sectores como la agricultura y la industria en muchas regiones brasileiras, como ya ocurre en muchos países.

El riego con efluentes sanitarios (agua servida tratada) puede ser considerada como un ferti-riego debiéndose por tanto, observar todos los cuidados respecto a tal práctica. Ya sea el descarte indebido de efluentes puede representar un problema ambiental, por otro lado presenta características deseables, como potenciales para el abastecimiento de nutrientes a las plantas, principalmente el nitrógeno, fósforo y potasio y, sobre todo, potencialidad de uso como fuente extra de agua. Kiziloglu et al. (2008) afirman que la utilización de aguas tratadas para la irrigación o riego es cada vez mayor, siendo una solución técnica para minimizar la degradación del suelo y para restaurar el contenido de nutrientes de los mismos. Segundo
Heidarpour et al. (2007), la creciente necesidad de agua en las regiones áridas del mundo, resultó en la necesidad del uso de aguas tratada para la aplicación en la agricultura y el paisajismo.

Con el desarrollo de la agricultura en las últimas décadas y con la necesidad de aumentar la productividad y la calidad de los productos, el agricultor necesitó tecnificar cada vez más, adecuando su producto y sus medios de producción para la demanda actual.

El uso de riego por goteo en cultivos regados con agua servida tratada ha 
sido aceptado entre agricultores e investigadores en varios partes del mundo, demostrando beneficios como el mejor control y requerimiento en la aplicación de agua y nutrientes; aumento en la producción agrícola y menor posibilidad de contaminación del cultivo. Asociado a ferti-riego, el sistema por gotas puede proporcionar incrementos de productividad de frutos y economía de agua en el orden de 10 a 30\%, en comparación a los sistemas por aspersión.

El tomate (Lycopersicum esculentum) es una de las hortalizas más significativas cultivadas en el Brasil (Luz et al., 2007) por tratarse de uno de los principales cultivos producidos en invernadero, resultando en frutos de excelente calidad comercial garantizado, así, como una ganancia mayor para los productores. Es una de las hortalizas de fruto más producidas en la región Centro-Oeste, siendo el Estado de Goiás el mayor productor, con una participación en el CEASA-GO el año 2006, siendo 84\% producidos en el mismo Estado, del total comercializado que fue de 7.599,90 t. La cantidad por variedad y porcentaje producida en Goiás fue: tomate Santa Cruz 1 (18.789,60 t, $84,14 \%)$, tomate Ensalada $1(2.452,41 \mathrm{t}$, 92,66\%), tomate Larga Vida (39.789,95 t, 78,02\%) y tomate Cereza (15.118 t, 97,64\%). Los principales Municipios productores son Anápolis (145,28 t de tomate Ensalada 1; 5.509,50 t de tomate Larga Vida y $1.539,08 \mathrm{t}$ de tomate Santa Cruz, totalizando 7.193,81 t), Ouro Verde, Bonfinópolis, Goianápolis, Leopoldo de Bulhões, Corumbá de Goiás, Vianópolis, Pirinópolis y Silvania (CEASA, 2007).

En la comercialización de hortalizas, un factor desfavorable es el alto índice de pierdas post-cosecha, que reducen sensiblemente la disponibilidad de ese producto (Magalhães et al., 2009).

La cultura "Dominador" es del tipo Larga Vida y es una de las más cultivadas y comercializadas en el país por dar mayor durabilidad después de la cosecha, alta resistencia a virus y tolerancia a bacterias, alta productividad y excelente padrón de frutos. Es la variedad más adaptada para el plantío en el verano porque mantiene el vigor en la planta con frutos uniformes y firmes (Fruticom, 2010).

Durante el período de 1990 a 2005 la producción de tomate (industrial y de mesa) el Brasil aumentó en 44,5\%, un área media cultivada en 60 mil hectáreas con una productividad que pasó de 37,1 mil kg/ha para $56,7 \mathrm{mil} \mathrm{kg} / \mathrm{ha}$. En el caso de tomates de mesa para consumo en natura, el volumen producido en 2004 fue de 2.128,4 mil toneladas, o sea $61 \%$ del total nacional (Camargo et al., 2006) citado por Bolzan (2008).

En muchas frutas, el equivalente entre los ácidos orgánicos y los azúcares es utilizado como criterio de evaluación del aroma. Con todo, como son algunos constituyentes volátiles, esa relación es más indicativa del sabor, porque se utiliza a acidez de titulación y no la acidez total, cuando se establece esa relación. Además de eso, algunos productos insípidos, conteniendo acidez y cantidad de sólidos solubles muy bajos, presenta una relación elevada entre eses componentes, lo que puede llevar a interpretación errónea de la calidad comercial. La relación aumenta con la madurez debido a la reducción en la acidez, hecho que permite una relación elevada, en frutas conteniendo bajo contenido de sólidos solubles (Chitarra \& Chitarra, 2005).

Los tomates cv. Diva, estudiados por Gomes \& Camelo (2002) almacenados en atmósfera controlada, presentaron una variación de $0,35 \%$ a $0,46 \%$ de acidez de titulación, en cuanto que la relación SS/AT fue de 11,85 e 16,05, indicando frutos de baja acidez y de buena calidad organoléptica.

Conociéndose el contenido de sólidos solubles (SS) y la acidez de titulación (AT) se puede establecer, para las frutas, la relación $\mathrm{SS} / \mathrm{AT}\left({ }^{\circ} \mathrm{Brix} / \%\right.$ de ácido). Alto valor de correlación indica una excelente combinación de azúcares y ácido que se correlacionan con sabor suave, en cuanto que los valores bajos, con sabor ácido.

Así, el objetivo fue evaluar la producción y la productividad del tomate cultivado en el interior y exterior del invernadero, utilizando agua tratada, agua de ferti-riego, agua natural y el desempeño del sistema de irrigación del tipo superficial y el sub-superficial en los tratamientos.

\section{MATERIAL Y MÉTODOS}

La investigación de producción de tomate fue realizada en la "Universidad Estadual de Goiás", Anápolis-GO (16 ${ }^{\circ} 19^{\prime} 36^{\prime \prime} \mathrm{S}, 48^{\circ} 57^{\prime} 10^{\prime}$ 'O, altitud de $1.017 \mathrm{~m}$ ), en la fecha del 21 de noviembre del 2007 al 20 de octubre del 2009. El clima es meso-térmico y húmedo clasificado como probable clima tropical de altitud, temperatura media anual de $22^{\circ} \mathrm{C}$, con bajas temperatura en junio $\mathrm{y}$ julio, media mínima de $10^{\circ} \mathrm{C}$.

El periodo de lluvia ocurre entre noviembre y marzo, con precipitación media de $1450 \mathrm{~mm}$. Las condiciones ambientales durante la conducción de los experimentos en el interior del invernadero y durante los meses de agosto y setiembre presentaron una humedad relativa media de $56,5 \%$, temperatura máxima de $34,1^{\circ} \mathrm{C}$ y temperatura mínima de $18,6^{\circ} \mathrm{C}$. En el exterior del invernadero para ese periodo la humedad media fue de $68,8 \%$, temperatura máxima de $29,5^{\circ} \mathrm{C}$ y temperatura mínima de $18,7^{\circ} \mathrm{C}$. Los datos climatológicos fueron registrados en el horario de las 11:00 horas.

Para el experimento fue seleccionado la variedad "Dominador" por ser una de las más comercializada y cultivada en la región de Anápolis-GO, entre las variedades de tomate ensalada. El delineamiento experimental fue de bloques al azar $3 \times 2$, siendo las parcelas constituidas por la calidad del agua y tipo de sistema de riego, con 4 repeticiones, resultando en los siguientes tratamientos: A1 I1) agua tratada y goteo superficial; A1 I2) agua tratada y goteo sub-superficial; A2 I1) ferti-riego convencional y goteo superficial; A2 I2) ferti-riego convencional y goteo subsuperficial; A3 I1) agua natural y goteo superficial; A3 I2) agua natural y goteo sub-superficial.

Sistema y manejo del riego Fueron utilizados tubos de goteo de 12 $\mathrm{mm}$ de diámetro, instalados a $0,05 \mathrm{~m}$ de la línea de plantas en el goteo superficial y enterradas a $0,15 \mathrm{~m}$ de la superficie del suelo sobre la línea de la planta en el goteo sub-superficial. Los emisores 
tenían un caudal nominal de $1,6 \mathrm{~L} / \mathrm{h}$, funcionando a una presión de $120 \mathrm{kPa}$, ajustada por medio de un regulador de presión en la entrada de las parcelas, con emisores a un espacio de $0,40 \mathrm{~m}$.

Para el manejo del riego en el interior del invernadero fue utilizado un mini-tanque de evaporación con medidas menores $(0,25 \mathrm{~m}$ de altura y 0,60 de diámetro) que el Tanque "Clase A", para determinar Et, siendo este valor corregido por un factor $\mathrm{Kp}$ (coeficiente de tanque) igual a 0,69 (Bernardo et al., 2006).

La lámina total del riego aplicada en el interior del invernadero fue de 141,90 $\mathrm{mm}$. y para el exterior del invernadero fue de 120,00 mm.

El agua tratada fue oriunda de la Estación de Tratamiento de aguas servidas de la Universidad Estadual de Goiás, (compuesto de 3 tanques asépticos modificadas en serie, con capacidad de 15000 L, 10000 L y 5000 L, seguidos de una laguna de Estabilización facultativa. El agua natural bien como el agua utilizada para ferti-riego fue obtenida de un pozo artesiano.

El suelo fue preparado a 20 días antes de la plantación, siendo realizado un arado mecanizado y posteriormente se realizó la nivelación manual del área, la recomendación para corrección de $\mathrm{pH}$ del suelo fue realizado conforme análisis del suelo: Profundidad (0-20), Ca (1,6), $\mathrm{Mg}(0,7), \mathrm{H}+\mathrm{Al}(3,1), \mathrm{K}(27), \mathrm{P}(1,8)$, MO (27), CTC $(5,5), \mathrm{V}(43,64 \%), \mathrm{Zn}$ $(0,8), \mathrm{B}(0,2), \mathrm{Na}(2,4), \mathrm{Cu}(0,4), \mathrm{Fe}$ $(57,3), \mathrm{S}(5,9), \mathrm{pH}(5,3)$,

Para el cultivo del tomatero, los nutrientes aplicados para los tres tratamientos en el transplante, cobertura y vía ferti-riego fueron: En el tratamiento con agua tratada más nutrientes granulados aplicados al esparcimiento en el suelo fue nitrógeno $400 \mathrm{~kg} / \mathrm{ha}$ (30\% en el transplante, $35 \%$ a los 25 DAT, y 35\% a los 45 DAT); $\mathrm{P}_{2} \mathrm{O}_{5} 1200 \mathrm{~kg} /$ ha en el transplante; $\mathrm{K}_{2} \mathrm{O}$ (50\% en el transplante, $30 \%$ a los 25 DAT, y 20\% a los 45 DAT); Zinc 4 kg/ ha en el transplante. En el tratamiento con agua natural y con nutrientes granulados aplicados al esparcimiento en el suelo la cantidad y la proporción fueron similares a los utilizados en el tratamiento anterior. Para el tratamiento con nutriente aplicados vía ferti-riego fue utilizando nitrógeno $400 \mathrm{~kg} / \mathrm{ha}(20 \%$ en el transplante y a los 25 DAT, 35\% a los 45 DAT y $25 \%$ a los 65 DAT); $\mathrm{P}_{2} \mathrm{O}_{5} 1200 \mathrm{~kg} / \mathrm{hay} \mathrm{K}_{2} \mathrm{O} 800 \mathrm{~kg} / \mathrm{ha}$ en la proporción $20 \%$ en el transplante y a los 25 DAT, $35 \%$ a los 45 DAT y $25 \%$ a los 65 DAT respectivamente; zinc $4 \mathrm{~kg} / \mathrm{ha}$ (20\% en el transplante y a los 25 DAT, $35 \%$ a los 45 DAT y $25 \%$ a los 6 DAT).

El manejo del riego fue realizado utilizando el mini-tanque Clase A y la humedad del suelo fue monitoreada a partir de la tensión matricial del suelo obtenida por medio de lectura de tensiómetros instalados en el suelo en las profundidades de 15 y $30 \mathrm{~cm}$ en cada uno de los tratamientos estudiados en el interior y exterior del invernadero.

En cada bloque fue instalado 16 tensiómetros tanto para tratamiento en el interior y exterior del invernadero. La lectura de tensión fue realizada con el uso de un tensímetro digital de punción y para cada valor de ese potencial matricial fue obtenida la humedad del suelo correspondiente, considerando la curva de retención de agua en el suelo, que fue obtenida a través de la colecta de muestras para el análisis física del suelo.

La cosecha delos tomates fue realizada considerando el punto de maduración fisiológica, que normalmente los productores consideran en la región de Anápolis-GO. El inicio de la cosecha ocurrió a los 95 días después del transplante (DAT) y se extendió hasta 110 DAT. Fueron transportados en bolsas de plástico sin refrigeración para el Laboratorio de la UEG, donde fueron mantenidos por un día a la temperatura y humedad relativa ambiente. Otra cantidad de tomates fue transportada para el laboratorio de la Embrapa Cerrados donde fueron mantenidos por 14 días en temperatura media de $27^{\circ} \mathrm{C}$ y humedad relativa de $60 \%$.

El análisis cuantitativo fue obtenida evaluándose el número total de frutos, rendimiento de frutos total, utilizando una balanza digital, marca Mettler PE 3600 , con precisión de 0,01 g. En el experimento se recogió 16 frutos por parcela experimental, totalizando 64 frutos por tratamiento, evaluando-se diámetros (DF) y altura de los frutos
(AL), utilizando-se vernier digital Mitutoyo Mod. CD-6"CSX-B con precisión de $0,1 \mathrm{~mm}$, y se calculó el índice de forma de los frutos (IFF), representado por la relación diámetro/ altura.

Se analizó en el inicio del almacenamiento, a los siete y catorce días de almacenamiento de los tomates (mantenidos a temperatura media de $27^{\circ} \mathrm{C}$ y humedad relativa de $60 \%$ ), $\mathrm{pH}$, sólidos solubles, acidez titulable, (relación) y azúcar soluble total. Para el análisis físico-químico de $\mathrm{pH}$, acidez titulable, sólidos solubles, y relación del mismo fueron realizadas de acuerdo con la metodología del Instituto Adolfo Lutz (2005). En la determinación de $\mathrm{pH}$ fueron colectadas aleatoriamente cuatro muestras de cada tratamiento, fue triturado y se realizó lecturas en un pHmetro digital Tecnal - TE C3-MP.

Para la determinación de los sólidos solubles ( ${ }^{\circ}$ Brix) fueron colocadas algunas gotas de pulpa homogenizada de cada muestra en la placa del refractómetro marca CETI NV - Quartz LCD 97801-EDM97, con precisión de $0,1 \%$. Para el análisis de la acidez titulable, fue titulado $10 \mathrm{~g}$ de pulpa homogenizada, diluida en $90 \mathrm{~mL}$ de agua destilada, con solución padrón de hidróxido de sodio $(\mathrm{NaOH})$ a $0,1 \mathrm{~N}$. Fue utilizado como punto de viraje a $\mathrm{pH} 8,1$, siendo el resultado expresado en gramos de ácido cítrico anhidro/100g.

Los azúcares solubles totales fueron determinados por el método de Antrona donde la reacción de antrona se basa en la acción hidrolítica y deshidratante del ácido sulfúrico concentrado sobre los carbohidratos. Cuando la reacción se lleva a efecto con los hidratos de carbono con ligaciones glicosídicas, estos son hidrolizadas y los azúcares simples deshidratados para furfural o hidroximetilfurfural. Esas substancias se condensan con la antrona (9.10-dihidro9-oxoantraceno) dando un producto de coloración azul petróleo. Los análisis fueron realizadas de acuerdo con el método propuesto por el Instituto Adolfo Lutz (2005). Fueron cuantificados por espectrofotometría a una longitud de onda de $620 \mathrm{~nm}$, utilizando una curva padrón de glicose, se utilizó el espectrofotómetro de Marca Thermo 
Fisher Scientific, modelo Biomate 3.

Para análisis estadística, los datos fueron sometidos para análisis de variancia, y las diferencias entre las medias, fueron comparadas por el teste de Tukey a 5\% de probabilidad. El análisis de regresión fue realizada a lo largo del tiempo de almacenamiento para cada tratamiento.

\section{RESULTADOS Y DISCUSION}

En la Tabla 1 se presenta los valores medios de la calidad del agua tratada y agua de pozo (natural) utilizadas en el riego del cultivo de tomatero en invernadero.

Se puede observar en la Tabla 1, que en el agua tratada se encuentra contenidos de nitrógeno-amoniaco $\left(\mathrm{N}-\mathrm{NH}_{4}\right)$ fueron en media de $20 \mathrm{mg} / \mathrm{L}$, considerados críticos para el riego de hortalizas (Trani, 2001), en cuanto en el agua de pozo (natural) no se detectó su presencia. Para Brasil (2005) en riego de hortalizas consumidas crudas el límite de nitrógeno amoniaco para $\mathrm{pH} \leq 7,5$, situación semejante de este trabajo, es de 3,5 mg/L, por tanto, los resultados obtenidos están encima de los padrones recomendados por esta resolución. Los valores normales de $\mathrm{N}-\mathrm{NH}_{4}^{+}$en el agua de riego están entre 0 y 5,0 mg/L (Ayers \& Westcot, 1991), más en el agua tratada doméstica o de las fábricas procesadoras de alimentos, están entre 10 y 50 $\mathrm{mg} / \mathrm{L}$. Para la grande mayoría de los cultivos, con excepción de las hortalizas, no ocurren efectos perjudiciales con concentraciones inferiores a $30 \mathrm{mg} / \mathrm{L}$, caso de este trabajo de investigación.

En la forma de nitrato en el agua tratada, el nitrógeno presentó valor medio de 0,29 mg/L. Para Ayers \& Weatcot (1991), en los valores de hasta 5 $\mathrm{mg} / \mathrm{L}$ de nitrato no existe restricción; de 5 a $30 \mathrm{mg} / \mathrm{L}$, a restricción es ligera a la moderada; ya para Trani (2001) y Brasil (2005), los valores límites de nitrato son de hasta $10,0 \mathrm{mg} / \mathrm{L}$ en el agua de riego para hortalizas, para que no ocurran problemas de salud a los consumidores de alimentos con exceso de nitrato y de contaminación de agua subterránea y superficial (Boink \& Speijers, 2001). Por sí solo, lo tóxico del nitrato en humanos es baja; en tanto, en 5 a 10\% del $\mathrm{NO}_{3}{ }^{-}$ingeridos en la alimentación es convertido a nitrito $\left(\mathrm{NO}_{2}^{-}\right)$en la saliva bucal o por reducción gastrointestinal; así, el nitrito, entrando en la corriente sanguínea, oxida el fierro $\left(\mathrm{Fe}^{2+} \rightarrow \mathrm{Fe}^{+}\right)$ de la hemoglobina, produciendo la metahemoglobina.

Los valores medios de fósforo total en el agua tratada fueron de $0,49 \mathrm{mg} / \mathrm{L}$ bastante inferior, a los encontrados por otros autores que observaron valor medio 9,4 mg/L en agua servida doméstico con tratamiento secundario. Estés contenidos, de acuerdo con Brasil (2005), están encima de lo permitido para la Clase 1, indicado para el riego de hortalizas consumidos en crudo, el cual permite un valor máximo de fósforo total de $0,025 \mathrm{mg} / \mathrm{L}$. Los valores críticos de fósforo en el agua de riego, según Trani (2001) son de $30 \mathrm{mg} / \mathrm{L}$; con todo, se observa grande disponibilidad entre las literaturas; dichas referencias pueden estar relacionadas a los objetivos del estudio de este parámetro en el agua, en cuanto para el agua de riego el máximo de fósforo debe ser definido de forma que no ocurra la formación de precipitados, como bicarbonato de calcio.

Los valores medios de potasio en el agua tratada fueron de 0,20 meq/L, no siendo lo suficiente para causar cualquier síntoma tóxico en el tomate; algunos autores encuentran una concentración media de potasio en el agua tratada de $0,64 \mathrm{meq} / \mathrm{L}$.

Tabla 1. Valores medios de la calidad del agua tratada y natural en el riego por goteo superficial y sub-superficial (mean values of wastewater quality and natural water used in drip irrigation and groundwater). Anápolis, UEG, 2011.

\begin{tabular}{|c|c|c|}
\hline Parámetros & Agua tratada & Agua de pozo \\
\hline Amoniaco $\mathrm{N}^{-\mathrm{NH}_{4}}{ }^{+}(\mathrm{mg} / \mathrm{L})$ & 20,00 & 0,00 \\
\hline Nitrato $\mathrm{N}^{-\mathrm{NO}_{3}}{ }^{-}(\mathrm{mg} / \mathrm{L})$ & 0,29 & 0,18 \\
\hline Fósforo total (mg/L) & 0,42 & 0,03 \\
\hline $\mathrm{P}_{2} \mathrm{O}_{5}(\mathrm{mg} / \mathrm{L})$ & 3,53 & 0,10 \\
\hline Potasio (meq/L) & 0,20 & 0,01 \\
\hline Calcio (meq/L) & 1,48 & 0,12 \\
\hline Sodio (meq/L) & 0,81 & 0,05 \\
\hline Magnesio (meq/L) & 0,21 & 0,01 \\
\hline $\operatorname{RAS}\left(\mathrm{mmol}_{\mathrm{c}} / \mathrm{L}\right)^{1 / 2}$ & 0,88 & 0,10 \\
\hline Conductivid eléctrica $(\mathrm{dS} / \mathrm{m})$ & 0,10 & 0,09 \\
\hline $\mathrm{pH}$ & 7,71 & 7,24 \\
\hline Azufre (mg/L) & 2,50 & 0,50 \\
\hline $\mathrm{DBO}(\mathrm{mg} / \mathrm{L})$ & 50,00 & 5,00 \\
\hline DQO $(\mathrm{mg} / \mathrm{L})$ & 180,00 & 14,00 \\
\hline Oxígeno disuelto $(\mathrm{mg} / \mathrm{L})$ & 2,71 & 3,22 \\
\hline Boro $(\mathrm{mg} / \mathrm{L})$ & 0,10 & $*$ \\
\hline Fierro $(\mathrm{mg} / \mathrm{L})$ & 0,50 & 0,50 \\
\hline Manganeso (mg/L) & $*$ & $*$ \\
\hline $\mathrm{SDT}^{1}(\mathrm{mg} / \mathrm{L})$ & 192,00 & 57,60 \\
\hline Sólidos sedimentables (mg/L) & $>4$ & $>1$ \\
\hline Sólidos Suspensos (mg/L) & 0,06 & 0,04 \\
\hline Sólidos Totales (mg/L) & 506,00 & 440,00 \\
\hline Dureza de calcio (mg/L) & 12,00 & 10,00 \\
\hline Dureza total (mg/L) & 11,89 & 3,60 \\
\hline Alcalinidad (mg/L) & 201,00 & 34,00 \\
\hline
\end{tabular}

RAS = relación de absorción de sodio (sodium absorption ratio); SDT= Total de sólidos disueltos (total dissolved solids); $\mathrm{DQO}=$ demanda química de oxígeno (chemical oxygen demand); Fuente: Paganini (1997), ${ }^{1} \mathrm{SDT}(\mathrm{mg} / \mathrm{L})=\mathrm{EC}(\mathrm{dS} / \mathrm{m}) \times 640$. 


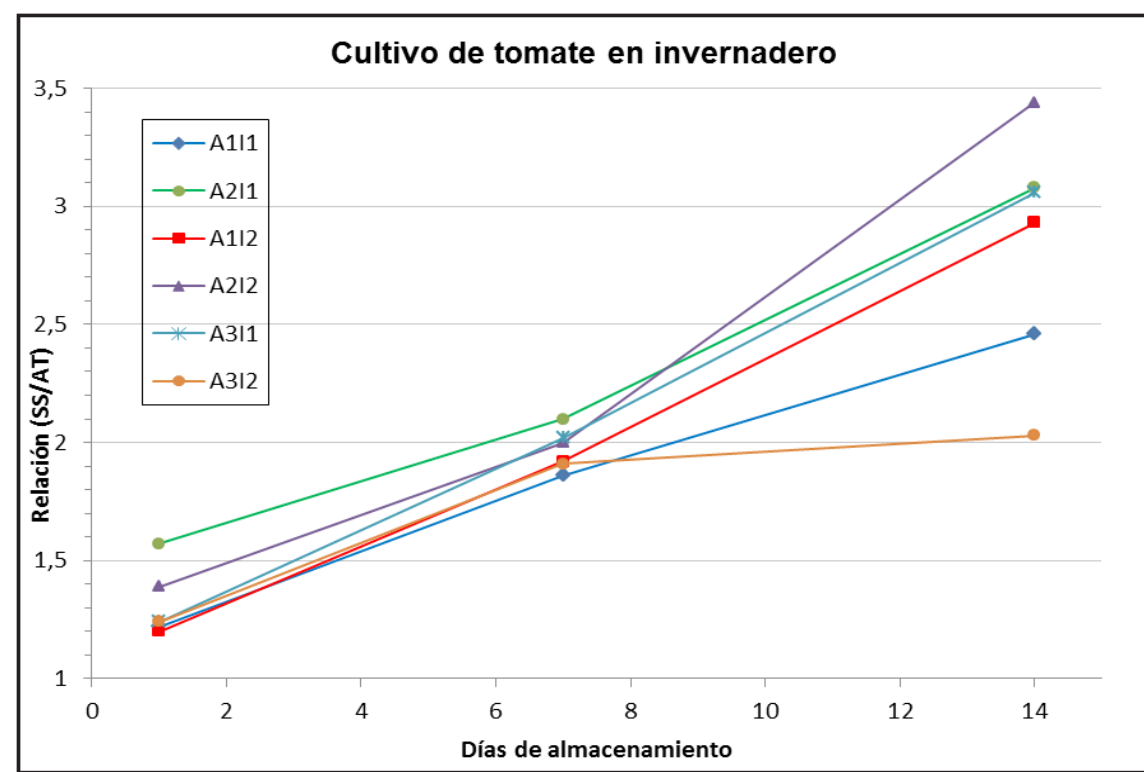

Figura 1. Relación (SS/AT) en frutos de tomatero en función del periodo de almacenamiento, cultivado en el interior del invernadero. [Ratio (SS/TA) in tomato depending on the storage period, grown inside the greenhouse]. Anápolis, UEG, 2011.

Considerando el contenido máximo de potasio, se tiene que el total adicionado al suelo vía agua tratada fue del orden de $15,5 \mathrm{~kg} / \mathrm{ha}$, se estima que cerca de 0,24 meq/L de $\mathrm{K}_{2} \mathrm{O}$ estuvieron presentes. En el agua de pozo la concentración media de potasio fue de $0,01 \mathrm{meq} / \mathrm{L}$, valores que son normales para el agua de riego que, de acuerdo con Ayers \& Westcot (1991), están entre 0 y $0,051 \mathrm{meq} / \mathrm{L}$.

El calcio indicó valores medios de $1,48 \mathrm{meq} / \mathrm{L}$ en el agua tratada $\mathrm{y}$, para el sodio, fue de $0,81 \mathrm{meq} / \mathrm{L}$; eses valores están bien bajos en los verificados por Medeiros et al. (2005) que obtienen valores medios de $1,83 \mathrm{meq} / \mathrm{L}, 0,69$ meq/L y 0,27 meq/L, para el Na, Ca y $\mathrm{Mg}$, respectivamente, en agua tratada con tratamientos secundarios. Considerando se las directrices presentados por Ayers $\&$ Westcot (1991), en que se refiere a la riego. Entretanto, no presentan síntomas de toxicidad a las plantas.

La razón máxima de absorción de sodio fue de $0,88\left(\mathrm{mmol}_{\mathrm{c}} / \mathrm{L}\right)^{1 / 2}$ (Tabla 1$)$ en el agua tratada y de $0,05\left(\mathrm{mmol}_{\mathrm{c}} / \mathrm{L}\right)^{1 / 2}$ para el agua de pozo. De acuerdo con Ayers \& Westcot (1991), para RAS hasta $3\left(\mathrm{mmol}_{\mathrm{c}} / \mathrm{L}\right)^{1 / 2}$, conjuntamente con EC menor que $0,7 \mathrm{dS} / \mathrm{m}$, no existe ningún grado de restricción de uso para el agua de riego, de esta forma, se observa que valores de sodio e RAS del agua tratada presentan debajo de lo normalmente encontrados en el agua tratada; se observa también que el agua con predominancia de los iones de sodio puede promover la disolución de las partículas de arcilla, disminuyendo la permeabilidad del suelo, causando reducción de la aireación del suelo e inhibición del desenvolvimiento del sistema radicular de las plantas con consecuente reducción de producción.

El valor de RAS fue de 0,88 $\left(\mathrm{mmol}_{\mathrm{c}} / \mathrm{L}\right)^{1 / 2}$ para el agua tratada y de 0,1 para el agua del depósito, inferior a los obtenidos por Jnad et al. (2001), en el efluente oriundo de cuatro localidades diferentes, variando entre 2,6 a 12,4 $\left(\mathrm{mmol}_{\mathrm{c}} / \mathrm{L}\right)^{1 / 2}$. Teniendo en vista a indicación de Ayers \& Westcot (1991).

La conductividad eléctrica media del agua $\left(\mathrm{Ce}_{\mathrm{a}}\right)$ tratada fue de $0,10 \mathrm{dS} / \mathrm{m}$. Considerándose la clasificación para el riego propuesto por Ayers \& Westcot (1991), el agua utilizada posee salinidad sin ningún grado de restricción, en que la $\mathrm{Ce}_{\mathrm{a}}$ puede llegar hasta $0,7 \mathrm{dS} / \mathrm{m}$ (450 mg/L de SDT), y ser utilizada en riego sin cualquier problema, hasta mismo para cultivos más sensibles a la salinidad.

El valor medio del $\mathrm{pH}$ en el agua tratada fue de $7,71 \mathrm{y}$, en el de pozo

Tabla 2. Productividad (t/ha) del cultivo de tomate "Dominador" en el interior y exterior del invernadero con diferentes tipos de agua y sistema de riego (tomato crop productivity "Dominador" inside and outside the greenhouse, irrigated with water and diferente types of irrigation systems). Anápolis, UEG, 2011.

\begin{tabular}{|c|c|c|c|c|c|c|c|c|}
\hline \multirow{2}{*}{ Tipo de agua } & \multicolumn{3}{|c|}{ Interior del invernadero } & \multirow{2}{*}{ Media } & \multicolumn{3}{|c|}{ Exterior del invernadero } & \multirow{2}{*}{ Media } \\
\hline & A1 & A2 & $\mathbf{A 3}$ & & A1 & A2 & A3 & \\
\hline I1 & $29,84 \mathrm{Aa}$ & $31,29 \mathrm{Aa}$ & $27,39 \mathrm{Aa}$ & 29,51 & $30,52 \mathrm{Aa}$ & $25,77 \mathrm{Aa}$ & $29,05 \mathrm{Aa}$ & 28,46 \\
\hline I2 & $32,50 \mathrm{Aa}$ & $31,92 \mathrm{Aa}$ & $25,34 \mathrm{Ab}$ & 29,92 & $28,95 \mathrm{Aa}$ & $39,45 \mathrm{Aa}$ & $26,58 \mathrm{Aa}$ & 31,66 \\
\hline
\end{tabular}

Regados con A1= Agua tratada (treated water); A2= ferti-riego convencional (conventional fertigation); $\mathrm{A} 3=$ agua natural (natural water); I1 = por goteo superficial (surface drip.); I2= por goteo sub-superficial (subsurface drip). Medias seguidas de las letras minúscula distintas en la línea y mayúsculas en la columna es diferente entre sí a nivel de 5\% indicado por el teste Tukey (average values followed by different lowercase letters on the line and uppercase letters in the columns differ from each other, Tukey $5 \%$ ). 
Tabla 3. Los valores medios de $\mathrm{pH}$, sólidos solubles y acidez titulable durante el almacenamiento en frutos de tomate producidos en el interior y exterior del invernadero, regados con diferentes tipos de agua y sistema de riego (Average values of pH, soluble solids and titratable acidity during storage of tomato fruits produced inside and outside the greenhouse, irrigated with different types of water and irrigation systems). Anápolis, UEG, 2011.

\begin{tabular}{|c|c|c|c|c|c|c|}
\hline \multirow{3}{*}{ Tratamiento } & \multicolumn{3}{|c|}{ Interior del invernadero } & \multicolumn{3}{|c|}{ Exterior del invernadero } \\
\hline & \multicolumn{6}{|c|}{ (días de almacenamiento) } \\
\hline & Zero & 07 & 14 & Zero & 07 & 14 \\
\hline & \multicolumn{6}{|c|}{ pH } \\
\hline A1 I1 & 3,97 a,a,B & $4,08 \mathrm{a}, \mathrm{a}, \mathrm{A}$ & $4,08 \mathrm{~b}, \mathrm{~b}, \mathrm{~B}$ & $4,08 \mathrm{a}, \mathrm{a}, \mathrm{A}$ & $4,11 \mathrm{a}, \mathrm{a}, \mathrm{A}$ & - \\
\hline A2 I1 & $4,15 \mathrm{a}, \mathrm{a}, \mathrm{A}$ & $4,21 \mathrm{a}, \mathrm{a}, \mathrm{A}$ & $4,25 \mathrm{a}, \mathrm{a}, \mathrm{A}$ & 4,19 a,a,A & $4,22 \mathrm{a}, \mathrm{a}, \mathrm{A}$ & $4,22 \mathrm{a},-, \mathrm{A}$ \\
\hline A3 I1 & 4,00 a,a,B & 4,16 a,a,A & 4,23 a,a,A & 4,04 a,a,C & 4,13 a,a,B & 4,20 a,a,A \\
\hline A1 I2 & 3,95 a,a,C & 4,11 a,a,B & $4,22 \mathrm{~b}, \mathrm{a}, \mathrm{A}$ & 4,01 a,a,A & 4,08 a,a,A & - \\
\hline A2 I2 & $4,15 \mathrm{a}, \mathrm{a}, \mathrm{B}$ & $4,21 \mathrm{a}, \mathrm{a}, \mathrm{B}$ & $4,31 \mathrm{a}, \mathrm{a}, \mathrm{A}$ & 4,11 a,a,A & $4,15 \mathrm{a}, \mathrm{a}, \mathrm{A}$ & - \\
\hline \multirow[t]{2}{*}{ A3 I2 } & $4,10 \mathrm{a}, \mathrm{a}, \mathrm{A}$ & $4,14 \mathrm{a}, \mathrm{a}, \mathrm{A}$ & $4,11 \mathrm{c}, \mathrm{b}, \mathrm{A}$ & 4,00 a,a,B & 4,03 a,a,B & $4,18-, \mathrm{a}, \mathrm{A}$ \\
\hline & \multicolumn{6}{|c|}{ Sólidos solubles ( ${ }^{\circ}$ Brix) } \\
\hline A1 I1 & $5,40 \mathrm{~b}, \mathrm{a}, \mathrm{C}$ & $6,75 \mathrm{a}, \mathrm{a}, \mathrm{B}$ & 8,00 a,a,A & 5,00 a,a,B & $6,00 \mathrm{a}, \mathrm{a}, \mathrm{A}$ & - \\
\hline A2 I1 & 5,95 a,a,B & $6,57 \mathrm{a}, \mathrm{a}, \mathrm{B}$ & $8,00 \mathrm{a}, \mathrm{a}, \mathrm{A}$ & $5,27 \mathrm{a}, \mathrm{a}, \mathrm{C}$ & $6,00 \mathrm{a}, \mathrm{a}, \mathrm{B}$ & $7,47 \mathrm{a},-, \mathrm{A}$ \\
\hline A3 I1 & 5,12 b,a,C & $6,70 \mathrm{a}, \mathrm{a}, \mathrm{B}$ & $7,77 \mathrm{a}, \mathrm{a}, \mathrm{A}$ & $5,12 \mathrm{a}, \mathrm{a}, \mathrm{C}$ & $6,00 \mathrm{a}, \mathrm{a}, \mathrm{B}$ & $7,57 \mathrm{a}, \mathrm{a}, \mathrm{A}$ \\
\hline A1 I2 & $4,62 \mathrm{a}, \mathrm{b}, \mathrm{C}$ & $6,82 \mathrm{a}, \mathrm{a}, \mathrm{B}$ & $8,00 \mathrm{a}, \mathrm{a}, \mathrm{A}$ & $5,45 \mathrm{a}, \mathrm{a}, \mathrm{B}$ & 6,12 a,a,A & - \\
\hline A2 I2 & $5,00 \mathrm{a}, \mathrm{b}, \mathrm{C}$ & $6,57 \mathrm{a}, \mathrm{a}, \mathrm{B}$ & 7,87 a,a,A & 5,07 a,a,A & $6,00 \mathrm{a}, \mathrm{a}, \mathrm{A}$ & -- \\
\hline \multirow[t]{2}{*}{ A3 I2 } & $5,05 \mathrm{a}, \mathrm{a}, \mathrm{C}$ & $6,57 \mathrm{a}, \mathrm{a}, \mathrm{B}$ & 7,72 a,a,A & $5,45 \mathrm{a}, \mathrm{a}, \mathrm{C}$ & $6,22 \mathrm{a}, \mathrm{a}, \mathrm{B}$ & $7,72-, \mathrm{a}, \mathrm{A}$ \\
\hline & \multicolumn{6}{|c|}{ Acidez titulable (g de ácido cítrico anhidro/100 g de muestra) } \\
\hline A1 I1 & $4,44 \mathrm{a}, \mathrm{a}, \mathrm{A}$ & 3,61 a,a,B & 3,26 a,a,B & $3,85 \mathrm{a}, \mathrm{a}, \mathrm{A}$ & 3,21 a,a,B & - \\
\hline A2 I1 & 3,85 a,a,A & $3,13 \mathrm{a}, \mathrm{a}, \mathrm{AB}$ & $2,60 \mathrm{a}, \mathrm{a}, \mathrm{B}$ & 3,25 a,a,A & 3,06 a,a,A & $2,74 \mathrm{a},-, \mathrm{A}$ \\
\hline A3 I1 & $4,15 \mathrm{a}, \mathrm{a}, \mathrm{A}$ & 3,33 a,a,AB & $2,55 \mathrm{a}, \mathrm{b}, \mathrm{B}$ & $3,62 \mathrm{a}, \mathrm{a}, \mathrm{A}$ & $3,34 \mathrm{a}, \mathrm{a}, \mathrm{B}$ & $2,85 \mathrm{a}, \mathrm{a}, \mathrm{AB}$ \\
\hline A1 I2 & 3,89 a,a,A & $3,55 \mathrm{a}, \mathrm{a}, \mathrm{AB}$ & 2,73 ab,a,B & $4,25 \mathrm{a}, \mathrm{a}, \mathrm{A}$ & 3,67 a,a,B & - \\
\hline A2 I2 & $3,63 \mathrm{a}, \mathrm{a}, \mathrm{A}$ & 3,29 a,a,A & 2,73 b,a,B & $3,41 \mathrm{a}, \mathrm{a}, \mathrm{A}$ & 3,11 a,a,A & - \\
\hline A3 I2 & 3,97 a,a,A & 3,42 a,a, $\mathrm{AB}$ & 3,80 a,a,B & 4,26 a,a,A & $3,53 \mathrm{a}, \mathrm{a}, \mathrm{AB}$ & $2,71-, \mathrm{a}, \mathrm{B}$ \\
\hline
\end{tabular}

Regados con A1 I1= agua tratada y goteo superficial (treated waste water and surface drip); A1 I2= agua tratada y goteo sub-superficial (treated waste water and sub-surface drip); A2 I1 = ferti-riego convencional y goteo superficial (fertigation and conventional surface drip); A2 I2 = ferti-riego convencional y goteo sub-superficial (conventional fertigation and subsurface drip); A3 I1= agua natural y goteo superficial (natural water and surface drip); A3 I2= agua natural y goteo sub-superficial (natural water and sub-surface drip). Media seguida de la misma letra minúscula en la primera columna no difiere entre los tipos de agua para el mismo sistema de riego, y en la segunda columna entre los tipos de riego para una misma calidad de agua; letra mayúscula igual en la línea no difiere entre sí, por el teste de Tukey a 5\% de probabilidad (mean values followed by the same lowercase letter in the first column do not differ between types of water for the same irrigation system, and in the second column between the types of irrigation for the same quality of water; same capital letter in the line do not differ from each other by the teste Tukey at 5\% probability). (-)= Tratamientos descartados por el análisis sensorial (treatments discarded by sensory analysis).

fue de 7,24, valores que están dentro del intervalo recomendado por Brasil (2005) para riego de hortalizas que deben estar entre 6,0 y 9,0 .

Los mayores valores de azufre fueron de 2,5 para el agua tratada y de $0,5 \mathrm{mg} / \mathrm{L}$ para el agua de pozo. En general, el azufre proviene de la utilización del sulfato de amonio como fuente de nitrógeno en las aplicaciones complementarias, ya que posee azufre en su formulación, por tanto, en el agua tratada el azufre presenta valores elevados, mas normalmente, no ocasiona cualquier tipo de problemas a las plantas.

Los valores de la demanda bioquímica de oxígeno DBO, observados se encuentran encima del máximo permitido para la clase 1 , que es de 3 mg/L (Brasil, 2005); se puede ver, por tanto, grande variación de la DBO entre las fechas de la tomada de muestras, en virtud de la calidad de los efluentes tratados, que presenta variaciones en función de la intensidad de uso de las dependencias de la Universidad, tanto durante los días de la semana como durante el periodo del día, en tanto de posibles variaciones de caudal de entrada en el sistema de tratamiento del efluente o agua servida y de las condiciones climáticas.

El contenido medio de boro en el agua tratada fue de $0,10 \mathrm{mg} / \mathrm{L}$, no presenta ninguna restricción para aplicación en el riego que, de acuerdo con Ayers \& Westcot (1991), puede haber problemas cuando la concentración alcance por lo menos a $0,7 \mathrm{mg} / \mathrm{L}$, en tanto para Trani (2001), los valores límites para el agua de riego están entre 0,5 a 1,0 mg/L. El 


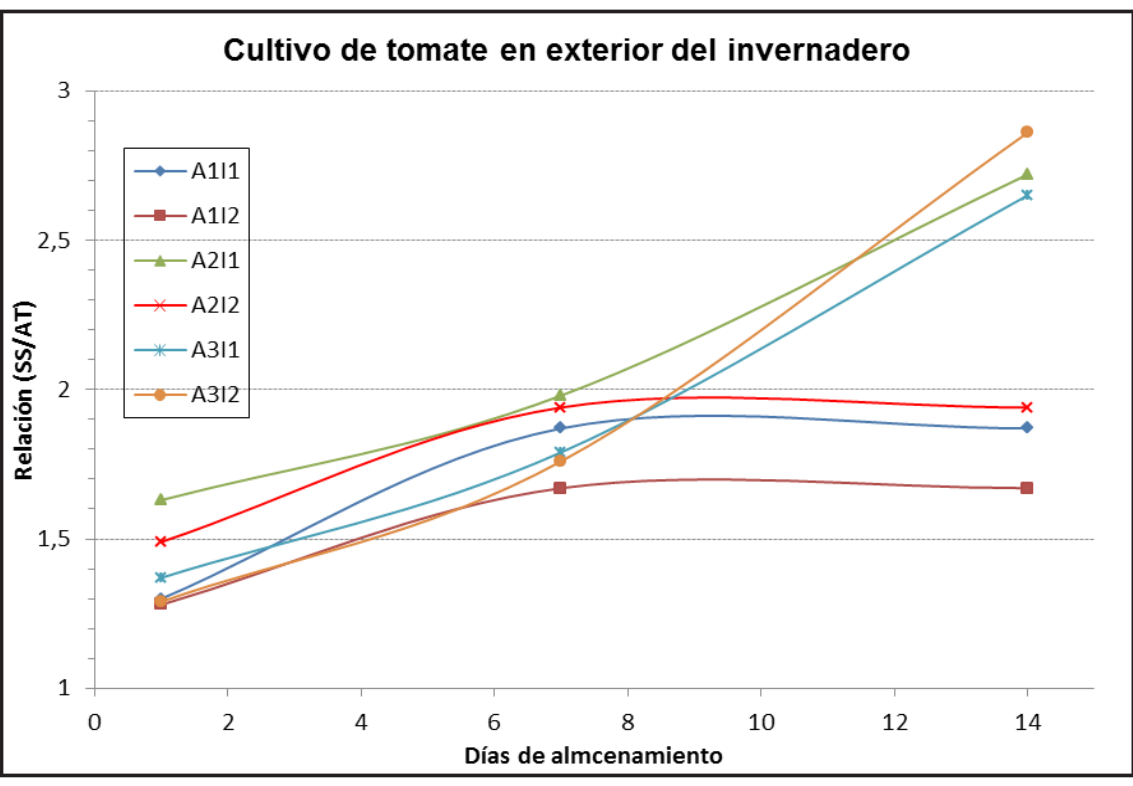

Figura 2. Relación (SS/AT) en frutos de tomatero en función del periodo de almacenamiento, cultivado en el exterior del invernadero. [Ratio (SS/TA) in tomato depending on the storage period, grown outside the greenhouse]. Anápolis UEG, 2011.

límite máximo para Brasil (2005) en el agua de riego de la clase 1 , es de 0,5 $\mathrm{mg} / \mathrm{L}$. Los límites recomendados de boro en el agua tratada utilizadas en el riego en el corto plazo, pueden ser de hasta de $2 \mathrm{mg} / \mathrm{L}$.

En el agua tratada y agua de pozo la concentración de fierro fue de $0,5 \mathrm{mg} / \mathrm{L}$. De acuerdo con Trani \& Carrijo (2004), los valores máximos de fierro en el agua de riego deben estar entre 0,2 a 1,5 $\mathrm{mg} / \mathrm{L}$, visto que, encima de este límite superior, pude ocurrir precipitación de $\mathrm{F}_{2} \mathrm{Cl}_{3}$ en aguas con altos contenidos de cloro. Los valores menores de fierro total deben ser utilizados en cultivos que sean consumidas en estado crudas, como indicado por Brasil (2005), que recomienda el límite máximo de 0,3 $\mathrm{mg} / \mathrm{L}$.

La media de la productividad de tomates para los tres tratamientos (agua tratada A1, ferti-riego convencional A2, agua natural A3) fue superior $(29,92 \mathrm{t} /$ ha) cuando el riego fue por goteo subsuperficial, I2 y comparado al riego por goteo superficial, I1 $(29,51 \mathrm{t} / \mathrm{ha})$ para el cultivo en el interior del invernadero (Tabla 2). Igualmente la media fue superior $(31,66 \mathrm{t} / \mathrm{ha})$ cuando el riego fue goteo sub-superficial (I2) comparado al riego por goteo superficial $(28,46 \mathrm{t} /$ ha) para los cultivos en el exterior del invernadero (Tabla 2).
La productividad en el tratamiento con agua tratada (A1) fue de 1,82\% mayor del que fue con ferti-riego convencional (A2) y de $25,97 \%$ mayor que el tratamiento con agua de testigo (A3) utilizando el riego sub-superficial (I2) en el interior del invernadero.

La productividad para el tratamiento con ferti-riego convencional y por goteo sub-superficial (A2I2), cultivado en el exterior del invernadero fue de $39,45 \mathrm{t} /$ ha, siendo así, está dentro de límite de la productividad (37,1 a 56,7 t/ha) como es indicado en el trabajo de Camargo (2006) citado por Bolzan (2008) y en el rango de 15,0 a 73,2 t/ha según Lima et al. (2011). Para el tratamiento (A2I2) fue de 31,92 t/ha, o sea, es menor a los indicados por el mismo autor anteriormente citado, con excepción de Lima et al. (2011).

Santos (2009), en una pesquisa con tomate "Dominador" obtiene una media en el diámetro de $71,7 \mathrm{~mm}$ y altura media de $58,8 \mathrm{~mm}$, resultando en una índice de formato del fruto IFF de 1,22, por tanto, poco debajo de lo obtenido en este trabajo de investigación, que la media fue de 1,3 para el cultivo en el interior del invernadero y 1,25 para el cultivo en el exterior del mismo. De acuerdo con Brasil (2002), los cultivos de tomate de mesa son clasificados de acuerdo al formato del fruto, en dos grupos: alargado, cuando el diámetro longitudinal es mayor del que el transversal, y redondo cuando el diámetro longitudinal es menor o igual al transversal. Por tanto en esta pesquisa, el tomate "Dominador" fue clasificado como redondo.

De acuerdo con la Tabla 3, los valores de $\mathrm{pH}$ de los frutos de tomate producidos en el interior del invernadero varían entre 3,95 y 4,32 y en los producidos en el exterior, varían entre 4,00 y 4,22 durante todo el periodo de almacenamiento, siendo el mayor valor observado a los catorce días de almacenamiento en los tomates producidos en el invernadero utilizando ferti-riego convencional y por goteo sub-superficial (A2I2). Esa variación es similar a los valores obtenidos por Camargo et al. (2000), confirmando la clasificación del tomate como un fruto ácido, por poseer $\mathrm{pH}$ menor que 4,5. El valor de $\mathrm{pH}$ puede variar de acuerdo con las condiciones de almacenamiento, influenciando en el sabor del producto final. Es deseable, en general, un $\mathrm{pH}$ inferior a 4,5 para reducir la proliferación de microorganismos en el producto.

Los valores de sólidos solubles en el tomate "Dominador" en esta pesquisa aumentaron durante el periodo de almacenamiento para todos los tratamientos como se puede observar en la Tabla 3. De acuerdo con Morgan (2004), valores de sólidos solubles encima de $5,0^{\circ}$ Brix indican frutos de tomate de alta calidad organoléptica, siendo que el aumento del contendido de sólidos solubles está relacionado con el sabor de los frutos, por ser esa fracción compuesta por ácidos y azúcares, dando, así, mayor valor de calidad al producto.

Según Mir \& Beaudry (2002), la reducción de los sólidos solubles es normal, siendo justificada por el elevado consumo de ese substrato para suplir la energía necesaria para reaccione metabólicas, pues los azúcares constituyen importante substrato respiratorio. En todos los tratamientos se da aumentos de sólidos solubles lo que es consecuencia de la transformación de las reservas acumuladas durante la formación y el desenvolvimiento de eses sólidos en azúcares solubles. Valores 
mayores de sólidos solubles pueden ser debido a la variabilidad de las muestras, o a la pierda de agua ocurrido durante el almacenamiento. Según Brackmann et al. (2008), algunos autores asocian el aumento de sólidos soluble con la deshidratación de los frutos y la concentración de los azúcares.

Algunos valores de sólidos solubles observados en este trabajo de investigación se sitúan en el rango de variación de los cultivos de tomatero que producen frutos conteniendo sólidos solubles variando de 5,0 a $8,0^{\circ}$ Brix, para todos los tratamientos en el interior del invernadero y la media de los sólidos solubles fue de $8,0^{\circ}$ Brix superior a los obtenidos en los tratamientos en el exterior del invernadero (media de $6,0^{\circ}$ Brix), considerando un producto de calidad superior alcanzado en el periodo del almacenamiento, siendo así, muy superior a los encontrados por Lima et al. (2011) trabajando con tomate italiano, y de Brackmann et al. (2007) que trabajó con la variedad "Cronus" en almacenamiento en diferentes temperaturas $\left(4,18\right.$ y $4,30^{\circ}$ Brix $)$. De acuerdo con Raupp et al. (2009) el contenido sólidos solubles en el fruto, además de ser una característica genérica del cultivo, es influenciado por el abono, temperatura y el riego.

Los valores de acidez de titulación para los tomates de este trabajo y para los tratamientos, se redujeron durante el periodo de almacenamiento como se puede observar en la Tabla 3 . Ese comportamiento corresponde a lo que sucede generalmente con productos vegetales cosechados, con excepción de frutas de caroso, como durazno, ciruela y otros, que presentan aumento en la acidez después de la cosecha, correlacionándose con la generación de radicales ácidos en la pared celular. De acuerdo con Chitarra \& Chitarra (2005), el contenido de ácidos orgánicos disminuye con la maduración de las frutas, en algunos casos, hay un pequeño aumento en los valores con el aumento de la maduración. También afirma que, después de la cosecha y durante el almacenamiento, la concentración total de ácidos orgánicos tiende a disminuir, siendo que los cambios post-cosecha varían con la especie de ácido en cuestión, tipo de tejido, manejo y condiciones de almacenamiento, cultivo, año de producción y diversas otras variables.

De esa forma se puede concluir que la disminución en la acidez se debe, probablemente, a la actividad metabólica más acentuada en los frutos de tomate mantenidos sobre condiciones de ambiente induciendo mayor consumo de ácidos orgánicos y radicales ácidos de la pared celular, pues la caída en la acidez puede en parte ser explicado por el consumo de moléculas ácidas, en especial los ácidos orgánicos en el proceso de respiración.

La relación sólidos soluble y acidez de titulación (SS/AT) es utilizada para indicar el equilibrio dulce-ácido de los alimentos. En ciertos frutos, si esta relación presenta grande desequilibrio, o sea, si la acidez disminuye mucho y aumenta los SS, puede ocurrir rechazo por parte de algunos consumidores.

Para la determinación de la relación de SS/AT fueron utilizados los resultados obtenidos para el contenido de sólidos soluble ( ${ }^{\circ}$ Brix) y acidez total de titulación ( $\%$ de ácido cítrico) de la Tabla 3, de una misma muestra, dividiendo los valores entre sí.

El valor en la relación $\mathrm{SS} / \mathrm{AT}$ indica una excelente combinación de azúcar y ácido que se correlaciona con el sabor suave de los frutos de tomate en los diferentes estadios de maduración (Ferreira, 2004).

Los valores de las SS/AT en este trabajo variaron entre 1,20 y 3,44 en los frutos de tomate producidos en el interior del invernadero y de 1,28 y 2,86 en los frutos producido en el exterior. El mayor valor $(3,44)$ de la relación fue observado en frutos producidos utilizando ferti-riego convencional y por goteo sub-superficial, esto puede haber ocurrido por la aplicación del riego que fue $141,90 \mathrm{~mm}$ para tratamientos en el interior del invernadero y de $120,00 \mathrm{~mm}$ para los tratamientos en el exterior del invernadero. En todos los tratamientos ocurrió aumento en esa variable durante el almacenamiento correspondiendo al aumento en los sólidos solubles y reducción de la acidez de titulación. De manera general, para obtenerse un balance sensorial equilibrado los valores de la relación deben estar entre 12 y 18 . Los valores obtenidos en ese trabajo en frutos de tomate "Dominador" para la variable fueron bien superiores a los valores citados por los autores, mostrando una calidad mucho superior.

La relación de sólidos solubles y acidez de titulación (SS/AT) se incrementan substancialmente desde el primer hasta los 14 días de almacenamiento a temperatura media de $27^{\circ} \mathrm{C}$ y a $60 \%$ de humedad relativa. En los tomates producidos en el interior del invernadero este incremento puede ser debido a los efectos encima indicado, o sea, incremento de sólidos solubles y reducción de acidez de titulación en el periodo del almacenamiento (Figura 1).

Estés resultados son esperados, pues las relaciones entre los atributos tienden ser de aumento de los valores de SS y disminución de los AT durante la senescencia de los frutos (Chitarra \& Chitarra, 2005). Resultados semejantes fueron obtenidos por Borguini (2002) y Ferreira (2004) citados por Bolzano (2008).

La relación de sólidos solubles y acidez de titulación (SS/AT) para los tomates producidos en el exterior del invernadero también se incrementó substancialmente desde el primer hasta los 14 días de almacenamiento a temperatura media de $27^{\circ} \mathrm{C}$ y a $60 \%$ de humedad relativa, excepto en los tomates de los tratamientos A1I1, A1I2 y A3I1 que no resistieron al almacenamiento hasta los 14 días, deteriorándose significativamente y los cuales fueron descartados definitivamente (Figura 2).

Con algunas excepciones para los frutos producidos en el exterior del invernadero, los valores de azúcares solubles totales se redujeron durante el almacenamiento. Los carbohidratos de menor peso molecular (azúcares mono y disacáridos) son compuestos sólidos generalmente solubles en agua y cristalizables el que puede llevar a la alteración de la consistencia y de la retención de agua en los alimentos. La mayoría tiene sabor dulce de intensidad variable conforme el azúcar (Bobbio \& Bobbio, 2001). En alimentos vegetales están presentes los azúcares y estés poseen papel importante en la caracterización del sabor y aroma por 
ser responsables por la dulzura. El contenido medio de los azúcares simples en hortalizas es baja y varían entre 2 y 5\% (Chitarra \& Chitarra, 2006), valores medios en este trabajo están en el rango indicados por el autor.

El tratamiento A3I2 presentó valor superior $(2,92 \%$ glucosa) comparado a los otros tratamiento cuando al contenido de azúcares solubles totales (AST) cultivados en el interior del invernadero. $\mathrm{Y}$ el cultivar A1I2 presentó valor superior $(3,69 \%$ glucosa $)$ en el contenido AST comparado a los otros tratamientos cultivados en el exterior del invernadero, mostrando un producto de calidad superior.

Los valores medios de azúcares solubles totales en el primer día de almacenamiento para todos los tratamientos en el interior y exterior del invernadero fueron superiores $(2,21$ a $3,69 \%$ glucosa), mas con el aumento del tiempo de almacenamiento, estés valores medios van cayendo significativamente hasta $0,42 \%$ de glucosa, excepto para algunos tratamientos como, por ejemplo, para A2I2 (cultivo en el exterior del invernadero) que tuvo un incremento desde 0,89 para $3,89 \%$ de glucosa.

\section{AGRADECIMIENTOS}

Los autores agradecemos al Programa Nacional de Post-Doctorado (PNDP), Coordenação de Aperfeiçoamento de Pessoal de Nível Superior (CAPES), Projeto No ${ }^{o}$ 1493/2008-UX-PE-PNPD $\mathrm{MEC} / \mathrm{CAPES}-\mathrm{MEC} / \mathrm{CNPq}-\mathrm{MEC} /$ FINEP, Brasil.

El autor Ricardo MV Valente agradece a la concesión de la bolsa pelo PVIV/UEG.

\section{REFERENCIAS}

AYERS RS; WESTCOT DW. 1991. A qualidade da água na agricultura. 2.ed. Campina Grande: UFPB. 218p. Estudos da FAO: Irrigação e Drenagem, 29 Revisado 1.

BERNARDO S; SOARES AA; MANTOVANI EC. 2006. Manual de Irrigação. 8.ed. Viçosa: UFV - Imprensa Universitária, 611p.

BOBBIO PA; BOBBIO F. O.2001 Química do processamento de alimentos. São Paulo: Varela
Editora e Livraria. 97p.

BOINK A; SPEIJERS G. 2001. Health effect of nitrates and nitrites, a review. Acta Horticulturae 563: 29-36.

BOLZAN RP. 2008. Biofilmes comestíveis para conservação pós-colheita de tomate 'Dominador'. Curitiba: UFPR. 167p (Tese mestrado).

BRACKMANN A; PETERLE ME; PINTO JAV; WEBER A; SAUTTER CK; EISERMANN AC. 2008. Temperatura e umidade relativa na qualidade da tangerina "Montenegrina" armazenada. Ciência Rural 38: 340-344.

BRACKMANN A; STEFFENS CA; ANDRIOLO JL; PINTO JAV. 2007. Armazenamento de tomate cultivar "Cronus" em função do estádio de maturação e da temperatura. Ciência Rural 37: $1295-1300$.

BRASIL. 2005. CONAMA, Resolução n 357. Dispõe sobre a classificação dos corpos de água e diretrizes ambientais para o seu enquadramento, bem como estabelece as condições e padrões de lançamento de efluentes. Diário Oficial da União, 17 de março de 2005.

BRASIL. 2002. Ministério da Agricultura, Pecuária e Abastecimento. Portaria SARC $\mathrm{n}^{\circ} 085$ de 06 de março de 2002. Propõe o Regulamento técnico de identidade e qualidade para classificação do tomate. Diário Oficial da União, Brasília, mar. 2002.

CAMARGO AMMP; CAMARGO FP; ALVES HS; CAMARGO FILHO WP. 2006. Desenvolvimento do sistema agroindustrial de tomate. Informações Econômicas In: BOLZAN RP (ed). Biofilmes comestíveis para conservação pós-colheita de tomate 'Dominador'. Curitiba: UFPR 36: 53-65. (Dissertação mestrado).

CAMARGO MI; FONTES PCR; FINGER FL; CARNICELLI JHA. 2000. Qualidade de tomate longa vida em estufa, influenciada por espaçamento e número de cachos por planta. Horticultura Brasileira 18: 562-563.

CEASA-GO. 2007. Acompanhamento conjuntural da comercialização. Disponível em: http:// www.ceasa.goias.gov.br. Acessado em: 08 de setembro de 2007.

CHITARRA MIF; CHITARRA AB. 2006. Pós-colheita de Frutas e Hortaliças. Lavras: UFLA. 256p.

CHITARRA MIF; CHITARRA AB. 2005. Póscolheita de frutos e hortaliças - fisiologia e manuseio. Lavras: Fundação de Apoio ao Ensino, Pesquisa e Extensão, 785 p.

FERREIRA SMR. 2004. Características de qualidade do tomate de mesa (Lycopersicum esculentum) cultivado nos sistemas convencional e orgânico comercializado na região metropolitana de Curitiba. Curitiba: UFPR. 231p (Tese doutorado).

FRUTICOM. 2010. O portal do mercado de frutas e cia: Multiplicação de variedades de tomate. Publicado em 27/09/2007. Disponível em: www.fruticom.com.br. Acessado em 10 de fevereiro de 2010.

GOMES FA; CAMELO AFL. 2002. Calidad postcosecha de tomates almacenados em atmosferas controladas. Horticultura
Brasileira 20: 38-43.

HEIDARPOUR M; MOSTAFAZADEH-FARD B; ABEDI KOUPAI J; MALEKIAN R. 2007. The effects of treated wastewater on soil chemical properties using subsurface and surface irrigation methods. Agricultural Water Management 90: 87-94.

INSTITUTO ADOLFO LUTZ. 2005. Normas Analíticas do Instituto Adolfo Lutz. 3.ed. São Paulo: Instituto Adolfo Lutz. 533p.

JNAD I; LESIKAR B; KENIMER A; SABBAGH G. 2001. Subsurface drip of residential effluent: I. soil chemical characteristics. Transaction of the ASAE 44: 1149-1157.

KIZILOGLU FM; TURAN M; SAHIN U; KUSLU Y; DURSUN A. 2008. Effects of untreated and treated wastewater irrigation on some chemical properties of cauliflower (Brassica olerecea var. botrytis) and red cabbage (Brassica olerecea var. rubra) grown on calcareous soil in Turkey. Agricultural Water Management 95: 716-724.

LIMA AA; ALVARENGA MAR; RODRIGUEZ L; CHITARRA AB. 2011. Yield and quality of tomato produced on substrates and with application of humic acids. Horticultura Brasileira 3: 269-274.

LUZ JMQ; SHINZATO AV; SILVA MAD. 2007. Comparação dos sistemas de produção de tomate convencional e orgânico em cultivo protegido. Bioscience Journal 23: 7-15.

MAGALHÃES AM; FERREIRAMD; MORETTI CL. 2009. Eficácia de limpeza durante o beneficiamento do tomate de mesa. Ciência Rural 39: 2431-2438.

MEDEIROS SS; SOARES AA; FERREIRA PA; NEVES JCL; MATOS AT; SOUZA JAA. 2005. Utilização de água residuária de origem doméstica na agricultura: Estudo das alterações químicas do solo. Revista Brasileira de Engenharia Agrícola e Ambiental 9: 603612.

MIR N; BEAUDRY R. 2002. Atmosphere control using oxygen and carbon dioxide. In: KNEE $\mathrm{M}(\mathrm{ed})$. Fruit quality and its biological basis. Columbus: Sheffield Academic. p.122-149.

MORGAN L. 2004. Tomato fruit flavor and quality evaluation. Part I. Disponível em: $<$ http:// www.fertcut.com/seach.cfm $>$ Acessado em 06 de março de 2010.

RAUPP DS. 2009. Processamento de tomate seco de diferentes cultivares. Acta Amazônica 39: 415-422.

SANTOS FFB. 2009. Obtenção e seleção de híbridos de tomate visando à resistência ao Tomato yellow vein streak virus (ToYVSV). Campinas: ATS-Genética. 86p (Tese mestrado).

TRANI PE; CARRIJO OA. 2004. Fertirrigação em hortaliças. Campinas: Instituto Agronômico, 58 p. (Boletim Técnico IAC, 196).

TRANI PE. 2001. Hortaliças folhosas e condimentos. In: PEREIRA ME; CRUZ MCP; VAN RAIJ B; ABREU CA (eds). Micronutrientes e elementos tóxicos na agricultura. Jaboticabal: CNPq/FAPESP/ POTAFOS, p.293-510. 